\title{
Laboratory studies with the systemic trichomonacide, metronidazole
}

\author{
R. F. JENNISON, P. STENTON, AND LESLIE WATT \\ From the Department of Clinical Pathology, Saint Mary's Hospitals for Women, \\ United Manchester Hospitals, Manchester
}

SYNOPSIS Laboratory studies with the trichomonacidal agent, metronidazole, show that 66 strains of $T$. vaginalis were killed in three days by concentrations of $0.0625 \mu \mathrm{g} . / \mathrm{ml}$. to $1 \mu \mathrm{g} . / \mathrm{ml}$.

Estimations of serum and urine levels in nine volunteers, the serum levels in 31 women, and the urine levels in 33 women show that metronidazole is rapidly absorbed and excreted in high concentration in the urine.

Development of resistance to metronidazole by $T$. vaginalis has not been demonstrated either in vivo or in vitro.

No toxic effect was demonstrated in the blood, liver, or kidney.

For some time it has been apparent that the effective treatment of infections due to $T$. vaginalis lies in a systematically administered drug. Aminitrazole was introduced in America (Plentl, Gray, Neslen, and Dalali, 1956) but whereas in common with many other compounds it showed considerable activity in vitro, it failed to produce blood levels and its use has been discontinued following unsuccessful trials (Catterall and Nicol, 1957; Dunlop, Philipp, and Watt, 1958; Jennison, 1957; Willcox, 1957).

In France, Cosar and Julou (1959) investigated metronidazole and found that it had a high antitrichomonal activity. Durel et al. (1960) showed that effective blood concentrations of this drug were obtained after oral administration and correlated this with cure of trichomoniasis and lack of toxicity. The efficacy of metronidazole has since been confirmed in Great Britain (Moffett and McGill, 1960; Rees, 1960; Watt and Jennison, 1960a).

Metronidazole has the formula 1-beta-hydroxyethyl-2-methyl-5-nitroimidazole. Its solubility in water is approximately $1 \%$, and in ethanol, ether, and chloroform approximately $0.5 \%$. The $p \mathrm{H}$ of a saturated aqueous solution at $20^{\circ} \mathrm{C}$. is about 5.8 . In the dosage used clinically it has no bactericidal activity and therefore does not interfere with normal vaginal flora. It has no antifungal activity.

The clinical trial of metronidazole reported by Watt and Jennison (1960a) continues, and the present paper describes some of the laboratory in-

Received for publication 10 October 1960. vestigations carried out during that trial. These investigations were directed towards elucidating the following points: (1) The sensitivity in vitro of various strains of $T$. vaginalis to metronidazole; (2) the possible development of acquired resistance to the drug in vitro or in vivo; (3) the levels of metronidazole in the blood and urine after ingestion of single doses by volunteers and after treatment with the recommended total dosage; (4) the possibility of toxic effects of the drug on the blood, liver, and kidneys.

\section{MATERIAL AND METHODS}

One hundred and eight women suffering from vaginal trichomoniasis have been treated with metronidazole as out-patients. The methods of diagnosis and tests of cure were as described in a previous paper (Watt and Jennison, $1960 \mathrm{a})$ and the length of observation varied from one to 23 weeks, with 60 patients $(55.5 \%)$ having been observed for 12 weeks or longer. All patients were treated with a standard course consisting of $600 \mathrm{mg}$. metronidazole daily for seven days. After treatment, $21(19.4 \%)$ have shown recurrence of $T$. vaginalis, but of these only four $(3.7 \%)$ showed $T$. vaginalis at the first examination immediately following the course of treatment. These four patients must be considered as failures. Eleven of the male consorts of these 21 patients have been examined and six $(54.5 \%)$ have been found to harbour $T$. vaginalis. The method used for demonstrating $T$. vaginalis in the male has been reported elsewhere (Watt and Jennison, 1960b).

The medium used for isolation of $T$. vaginalis was described by Stenton (1957), except that if monilia were seen in direct examination nystatin was added to the 
medium for primary culture. The same medium, omitting antibiotics in case of possible synergistic action, was used in the estimation of blood and urine levels of metronidazole and the sensitivity of $T$. vaginalis to that drug.

The stock strain of $T$. vaginalis used throughout these investigations had been isolated about seven months previously. This stock strain was maintained by subculturing $0.1 \mathrm{ml}$. of culture into $10 \mathrm{ml}$. of fresh medium every three days. It was considered that by subculturing frequently and using a measured inoculum of $T$. vaginalis standardization could be maintained throughout the investigation. The sensitivity of the stock strain was determined at first biweekly and later at weekly intervals. After 40 subcultures no change in sensitivity was noted.

Trichomonacidal levels of metronidazole for the various strains of $T$. vaginalis were estimated by subculturing $0.1 \mathrm{ml}$. of a three-day growth of culture into $10 \mathrm{ml}$. of Stenton's medium containing serial dilutions of metronidazole and incubating for three days at $37^{\circ} \mathrm{C}$. The cultures were then examined microscopically for living protozoa. Serum and urine levels were similarly determined by inoculating the stock strain of $T$. vaginalis in the ratio of $1: 100$ into medium containing dilutions of serum and urine. By comparison with the sensitivity of the stock strain the amount of metronidazole in serum or urine could be estimated.

\section{RESULTS OF INVESTIGATIONS}

SENSITIVITY OF T. VAGINALIS TO METRONIDAZOLE Sixty-six strains of $T$. vaginalis were tested for sensitivity to metronidazole; 55 of these were from

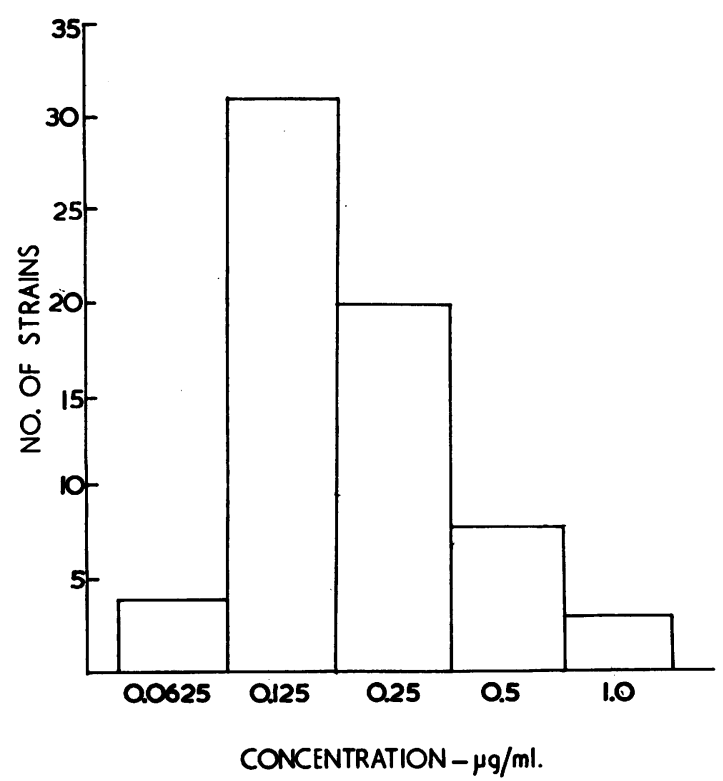

FIG. 1. Trichomonacidal levels of metronidazole for 66 strains of $\mathrm{T}$. vaginalis. women before treatment, seven were from women who did not respond to treatment or in whom the infection recurred later, and four were strains from male consorts.

Trichomonacidal levels varied from $1 \mu \mathrm{g}$. $/ \mathrm{ml}$. to $0.075 \mu \mathrm{g} . / \mathrm{ml}$. but $51(77 \%)$ of the strains were sensitive in the range of $0.25 \mu \mathrm{g} . / \mathrm{ml}$. to $0.125 \mu \mathrm{g} . / \mathrm{ml}$. (Fig. 1).

ACQUIRED RESISTANCE The strains isolated from patients who did not respond to treatment did not show any increased resistance to the drug.

An attempt to induce resistance in vitro was made by repeated subcultures from the immediate? sublethal dilution. It was found, however, that $\vec{f}$ the sublethal dose was trichomonacidal when the organisms were exposed to it for longer than three days, e.g., five days. Thus if cultures were incubated longer the organisms were sensitive to a lower concentration of metronidazole. Sub- $\vec{c}$

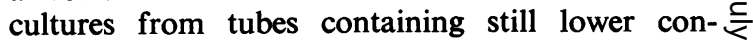
centrations of metronidazole also showed that $\vec{\oplus}$ prolonged exposure had a trichomonacidal effect. The method of subculturing from the immediate sublethal dilution is therefore not applicable in testing for the development of resistance to this drug in vitro.

SERUM AND URINE LEVELS These were estimated after a single dose and after a standard course.

After a single dose of $200 \mathrm{mg}$. A single dose of $200 \mathrm{mg}$. of metronidazole was given to nine volunteers and blood and urine samples collected at hourly intervals for four hours. In all subjects serum and urine after one hour showed trichomonacidal activity greatly in excess of the level required to inhibit the most resistant strain of $T$. vaginalis (Figs. $\frac{O}{2}$ 2 and 3 ). The peak was reached in most cases between two and three hours and thereafter the trichomon- $\frac{\text { o }}{9}$ acidal activity diminished.

After a standard course of treatment All patients were treated as out-patients so it was impossible to collect specimens at a standard time. Specimens were collected when the patients attended on the last day 0 of the course of treatment and the interval since $\omega$ taking the last $200 \mathrm{mg}$. tablet was recorded.

The serum and urine levels were estimated and are shown in Figs. 4 and 5. The levels obtained in $\varnothing$ patients who had not responded to treatment or who relapsed later are indicated. There is no evidence to $\underline{T}$ suggest defective absorption of metronidazole in $\frac{0}{\mathbb{D}}$ these patients.

In one patient who was lactating the level of $\stackrel{\mathbb{D}}{\square}$ metronidazole in the breast milk corresponded exactly to the serum level. 
FIG. 2. Composite graph drawn from estimations of trichomonacidal activity in the serum of nine normal subjects after the ingestion of $200 \mathrm{mg}$. of metronidazole.

FIG. 3. Composite graph drawn from estimations of trichomonacidal activity in the urine of nine normal subjects after the ingestion of $200 \mathrm{mg}$. of metronidazole.
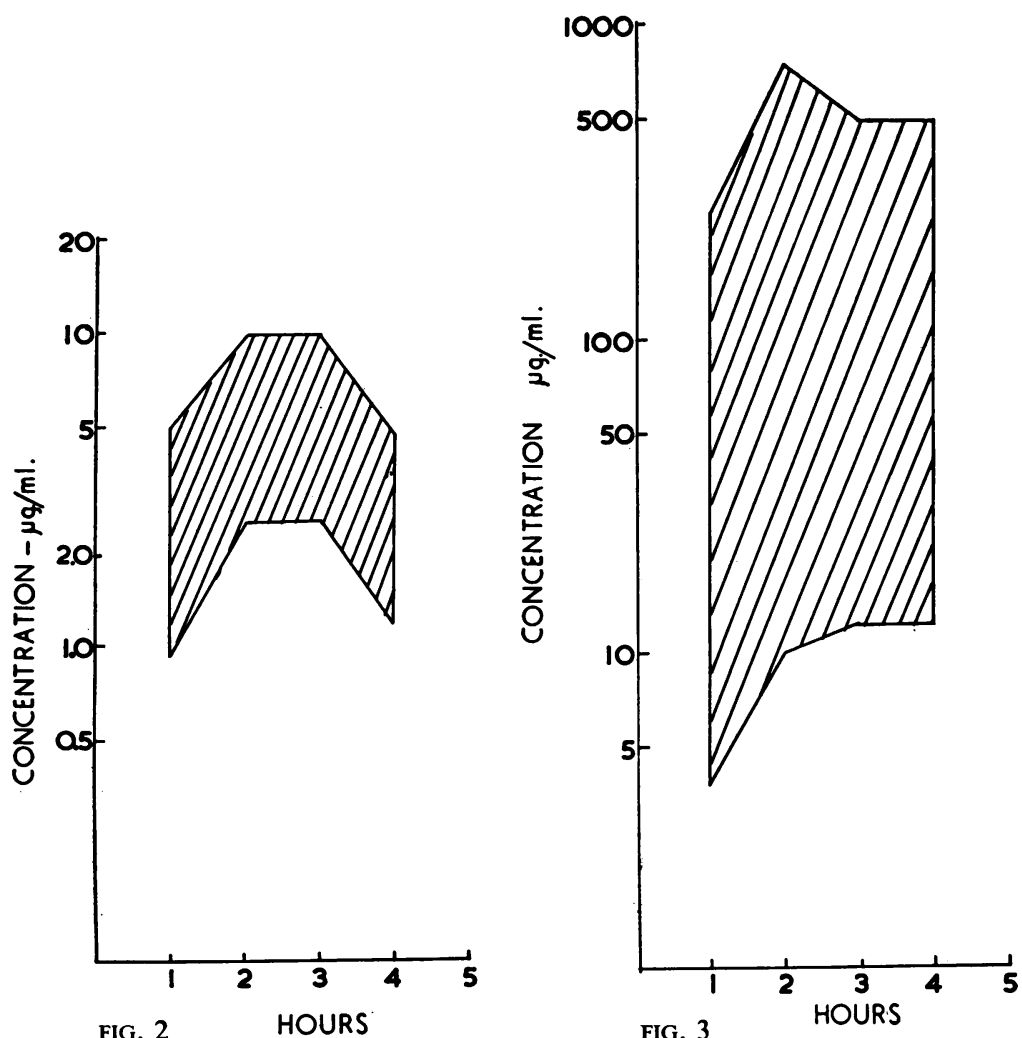

FIG. 3
TOXIC EFFECTS Because of the nitro group in metronidazole the possibility of toxic effects in the haemopoietic system or liver was considered. The possibility of renal damage was also examined because of the high urinary concentration of the drug.

Haemoglobin estimations, red cell, total and differential white cell, and platelet counts were carried out before and after treatment in eight patients and at various times up to three months after treatment in 48 patients. No significant change in the blood picture was noted.

Serum alkaline phosphatase and serum glutamic pyruvic transaminase were estimated in 12 patients after completion of the course of treatment but no increase in values occurred.

In most patients the urine was examined for albumin, cells, and casts but no abnormality was detected.

\section{DISCUSSION}

Durel, Roiron, Siboulet, and Borel (1960), using the method described by Bushby and Copp (1955), found that the inhibitory level of metronidazole was $1: 400,000$ in vitro. This is based on the $99 \%$ death rate at 24 hours, and, using this method, we demonstrated inhibitory levels between $1: 300,000$ and $1: 500,000$ in six strains. But the method used throughout this investigation differs in that, since three days is required for maximum growth in the medium used, the concentration of the drug in which no growth had occurred at the end of three days' incubation was taken as the trichomonacidal level. This method also more closely simulates the conditions in a patient undergoing treatment, both as regards sensitivity of the strain of trichomonad and the blood levels. The longer incubation caused inhibition at concentrations which would have allowed growth after 24 hours and by this method trichomonacidal levels have been found to vary from $1: 1,000,000$ to $1: 13,300,000(1 \mu \mathrm{g} . / \mathrm{ml}$. to $0.075 \mu \mathrm{g} . / \mathrm{ml}$.).

The inverse relationship between time and drug concentration was also apparent in the experiments designed to induce resistance in vitro. This is the converse of the effect of nystatin on $C$. albicans where the yeasts grow in higher concentrations of the antibiotic following prolonged incubation (Jennison and Stenton, 1957). 
The blood and urine estimations show that metronidazole is rapidly absorbed and rapidly excreted and concentrated by the kidney. The blood levels are maintained at an adequate level for some hours. Comparison of the blood and urine levels with the strain sensitivities shows that even the most resistant trichomonad should have been eliminated in every patient. At least five times the trichomonacidal level was maintained in the blood during the 12 hours after treatment ceased, and in the urine the lowest ratio in an individual patient was $200: 1$. It seems therefore that in those patients who did not respond to treatment this was due to failure of metronidazole to reach a nidus of infection rather than to a resistant strain or to failure to absorb the drug. The experimental relationship between concentration of metronidazole and time of incubation suggests that prolonging the course of treatment rather than increasing the dose might be more successful in these patients. Reinfection probably plays an important part in late recurrences. All except one of the strains isolated from consorts had the same drug sensitivity as that from his female partner. The one strain with a different sen-

FIG. 4. Trichomonacidal activity of serum in 31 patients after completion of a course of treatment with metronidazole. ( $X$ indicates patients who did not respond to treatment.)

FIG. 5. Trichomonacidal activity of urine in 33 patients after completion of a course of treatment with metronidazole. ( $X$ indicates patients who did not respond to treatment.)

FIG. 4 sitivity was in fact more sensitive, suggesting the existence of separate strains.

That metronidazole can act rapidly was shown in two in-patients who were not included in the clinical trial. Daily examination showed that trichomonads had disappeared in 24 hours in one patient and within 48 hours in the other.

There is no evidence to suggest that any resistance developed to metronidazole. Four strains isolated
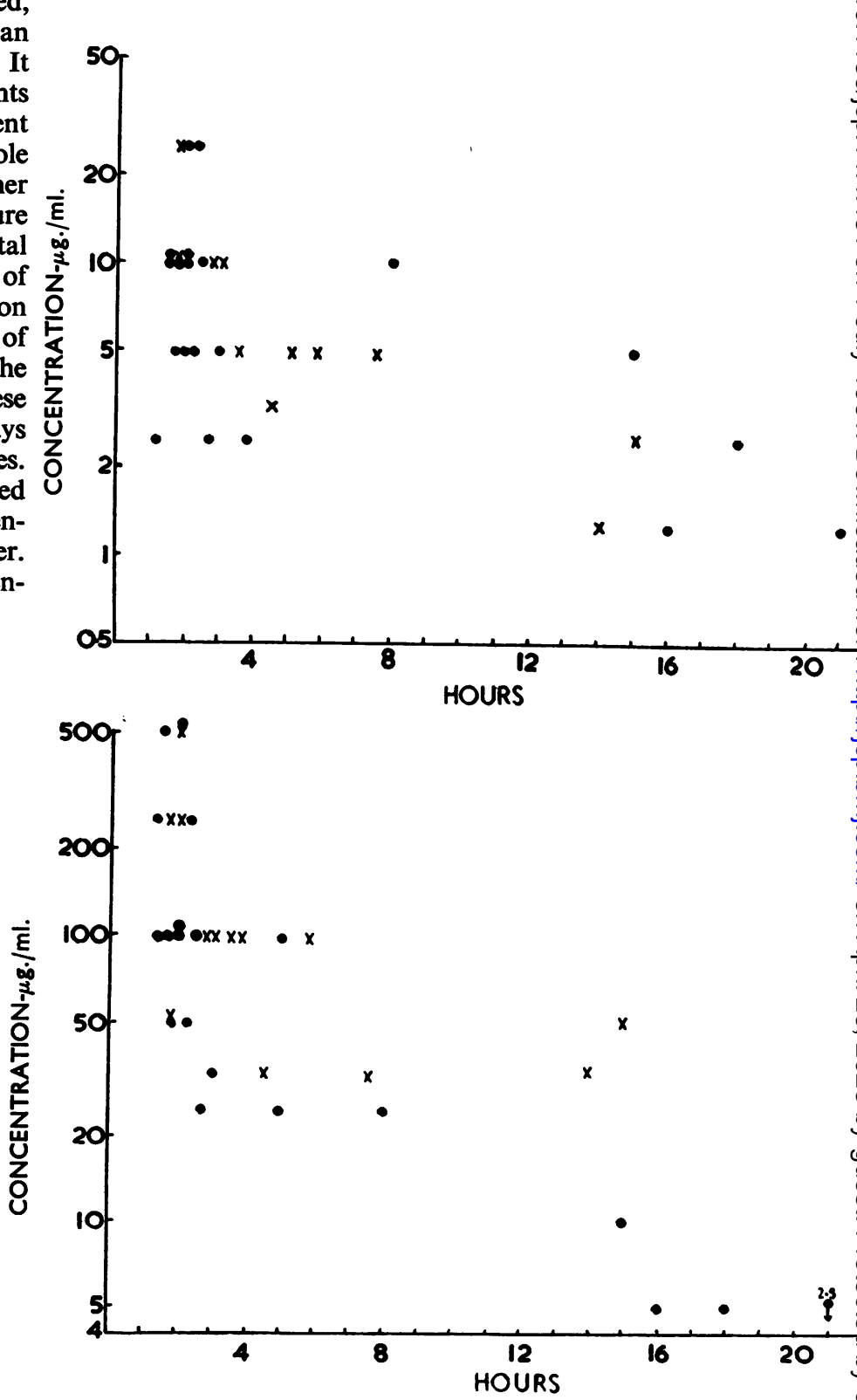
after treatment from patients who had not responded had exactly the same sensitivity as the strain isolated before treatment.

It is also of interest to note that the sensitivity of the stock strain did not vary after $\mathbf{4 0}$ subcultures in medium without the drug being added.

Approximately $20 \%$ of patients treated with metronidazole reported minor side-effects (Watt and Jennison, 1960a), but examination of the cellular elements of the blood, liver function tests, and urine analysis revealed no abnormality. These investigations have not demonstrated any toxic effect due to metronidazole but final assessment must await more extensive use of the drug.

We wish to thank the consultant gynaecological staff of Saint Mary's Hospitals for their cooperation, and Dr. R.
Forgan of May and Baker Ltd., who arranged a generous supply of metronidazole (Flagyl).

\section{REFERENCES}

Bushby, S. R. M., and Copp, F. C. (1955). J. Pharm. Pharmacol., 7, 112 .

Catterall, R. D., and Nicol, C. S. (1957). Brit. med. J., 2, 29.

Cosar, C., and Julou, L. (1959). Ann. Inst. Pasteur, 96, 238.

Dunlop, E. M. C., Philipp, E., and Watt, J. D. (1958). Brit. J. vener. Dis., 34, 57.

Durel, P., Roiron, V., Siboulet, A., and Borel, L. J. (1960). Ibid., 36, 21.

Jennison, R. F. (1957). Brit. med. J., 1, 517.

, and Stenton, P. (1957). J. clin. Path., 10, 219.

Moffett, M., and McGill, M. I. (1960). Brit. med. J., 2, 910.

Plentl, A. A., Gray, M. J., Neslen, E. D., and Dalali, S. J. (1956). Amer. J. Obstet. Gynec., 71, 116.

Rees, E. (1960). Brit. med. J., 2, 906.

Stenton, P. (1957). J. med. Lab. Technol., 14, 228

Watt, L., and Jennison, R. F. (1960a). Brit. med. J., 2, 902.

(1960b). Brit. J. vener. Dis., 36, 163.

Willcox, R. R. (1957). Ibid., 33, 115 